Automatic Black-Box Model Order Reduction using Radial Basis Functions

M. B. Stephanson, J. F. Lee, D. A. White

July 20, 2011

IEEE Antennas and Propagation Society

Spokane, WA, United States

July 4, 2001 through July 8, 2001 
This document was prepared as an account of work sponsored by an agency of the United States government. Neither the United States government nor Lawrence Livermore National Security, LLC, nor any of their employees makes any warranty, expressed or implied, or assumes any legal liability or responsibility for the accuracy, completeness, or usefulness of any information, apparatus, product, or process disclosed, or represents that its use would not infringe privately owned rights. Reference herein to any specific commercial product, process, or service by trade name, trademark, manufacturer, or otherwise does not necessarily constitute or imply its endorsement, recommendation, or favoring by the United States government or Lawrence Livermore National Security, LLC. The views and opinions of authors expressed herein do not necessarily state or reflect those of the United States government or Lawrence Livermore National Security, LLC, and shall not be used for advertising or product endorsement purposes. 


\section{Automatic, Black-Box Model Order Reduction Using Radial Basis Functions}

\author{
Matthew B. Stephanson* and Jin-Fa Lee \\ ElectroScience Laboratory \\ Department of Electrical and Computer Engineering \\ The Ohio State University, Columbus, Ohio 43201 \\ Email: stephanson.2@buckeyemail.osu.edu
}

\author{
Dan A. White \\ Computational Electromagnetics Group \\ Lawrence Livermore National Laboratory \\ Livermore, CA 94550
}

\begin{abstract}
Finite elements methods have long made use of model order reduction (MOR), particularly in the context of fast freqeucny sweeps. In this paper, we discuss a black-box MOR technique, applicable to a many solution methods and not restricted only to spectral responses. We also discuss automated methods for generating a reduced order model that meets a given error tolerance. Numerical examples demonstrate the effectiveness and wide applicability of the method.
\end{abstract}

\section{INTRODUCTION}

With the advent of improved computing hardware and numerous fast solution techniques, the field of computational electromagnetics are progressed rapidly in terms of the size and complexity of problems that can be solved. Numerous applications, however, require the solution of a problem for many different configurations, including optimization, parameter exploration, and uncertainly quantification, where the parameters that may be changed include frequency, material properties, geometric dimensions, etc. In such cases, thousands of solutions may be needed, so solve times of even a few minutes can be burdensome. Model order reduction (MOR) may alleviate this difficulty by creating a small model that can be evaluated quickly.

Many MOR techniques have been applied to electromagnetic problems over the past few decades, particularly in the context of fast frequency sweeps. Recent works have extended these methods to allow more than one parameter and to allow the parameters to represent material and geometric properties [1]. There are still limitations with these methods, however. First, they almost always assume that the finite element method is used to solve the problem, so that the system matrix is a known function of the parameters. Second, although some authors have presented adaptive methods (e.g., [2]), the order of the model is often determined before the MOR process begins, with little insight about what order is actually needed to reach the desired accuracy. Finally, it not clear how to efficiently extend most methods to the multiparameter case.

This paper address the above shortcomings be developing a method that uses a block-box approach to the solution method, is adaptive, and is easily extensible to many parameters.

This work perrformed under the auspices of the U.S. Department of Energy by Lawrence Livermore National Laboratory under Contract DEAC52-07NA27344.

\section{Model ORder Reduction PRELIMINARIES}

In this section we give a background explanation of the MOR method. To begin, we assume the particularly EM solver being used can be cast as an $N \times N$ matrix equation

$$
A(\mathbf{s}) \mathbf{x}(\mathbf{s})=\mathbf{b}(\mathbf{s}),
$$

where $\mathbf{s}$ is a vector of parameter values. To make development as general as we can, we assume that, given $\mathbf{s}$, the only operations that can be performed are solving for $\mathbf{x}(\mathbf{s})$, multiplying $A(\mathbf{s})$ with an arbitrary vector, and evaluating $\mathbf{b}(\mathbf{s})$. We note, in particular, that this assumption would make our framework applicable to algorithms such as the fast multipole method. Now, instead of solving this problem for every desired values of $\mathbf{s}$, we choose a reduced-dimension set of basis and testing functions, represented by the $N \times m$ matrices $V$ and $W$, respectively. This allows the reduced-order model to be formed,

$$
\tilde{A}(\mathbf{s})=W^{H} A(\mathbf{s}) V \tilde{\mathbf{x}}=\tilde{\mathbf{b}}(\mathbf{s})=W^{H} \mathbf{b}(\mathbf{s}) .
$$

In this work, we use a Galerkin-type approach and choose $W=V$. As a result of these projections, we now must solve a linear system with the $m \times m$ matrix $\tilde{A}$. If $m \ll N$, then this system can be solved much more rapidly than the original system. Once the model solution $\tilde{\mathrm{x}}$ is found, the true solution can be approximated as $\mathbf{x} \approx V \tilde{\mathbf{x}}$.

\section{Reduced Order Matrix Evaluation}

We first turn our attention to the problem of evaluating $\tilde{A}(\mathbf{s})$. In general, it is not possible to write $A(\mathbf{s})$ as a simple function of the parameters. And, for the sake of generality, we have expressly disclaimed such knowledge. Finally, it would not be efficient to simply calculate $V^{H} A V$ exactly for any $\mathrm{s}$, because the process of calculating $A$ and doing a matrixvector multiplication may be expensive. Even a few seconds would be too long, since we desire the reduced-order model to be evaluated very rapidly.

What can be done is to evaluate $V^{H} A V$ exactly for certain values of $\mathbf{s}$ and then interpolate the individual matrix entries. For although the solution currents or fields may be badly behaved, becoming singular at PEC corners, for example, the 
system matrix $A$ is typically bounded and continuous. Thus, we have the first ingredient of the method,

$$
\widehat{A}(\mathbf{s})=\sum_{i=1}^{M} f_{i}(\mathbf{s}) \tilde{A}_{i},
$$

with the condition that

$$
\widehat{A}\left(\mathbf{s}_{j}\right)=\tilde{A}\left(\mathbf{s}_{j}\right)
$$

at the parameter values $\left\{\mathbf{s}_{0}, \mathbf{s}_{1}, \ldots, \mathbf{s}_{M}\right\}$. Traditionally the $f_{i}$ have been monomials of the parameters, but we relax this to allow any set of functions that has suitable interpolatory properties.

\section{A. Radial Basis Function Interpolation}

Our choice of interpolating functions is motivated by two goals: easily handling large-dimensional $\mathbf{s}$ and the ability to build up the interpolation one sample at a time. Radial basis functions (RBFs) are well-suited for this task. They consist of any function of the form

$$
\Phi(\|\mathbf{s}-\mathbf{c}\|),
$$

where $\Phi$ is a scalar function and $\mathbf{c}$ is a constant vector with the same dimension as $\mathbf{s}$ [3]. One immediately notes that because the function depends only on the distance between $\mathbf{s}$ and $\mathbf{c}$, there is no difficulty with high dimensions. Also, a new RBF can be generated simply by choosing a new value of c. Common choices for $\Phi(r)$ include

$$
\begin{gathered}
\sqrt{r^{2}+a^{2}} \\
e^{-a r^{2}} \\
r^{2} \log r .
\end{gathered}
$$

Equation (6c) is part of a family of RBFs, having the form

$$
r^{2 p} \log r,
$$

where $p$ is a positive integer. When these RBFs are supplemented with polynomials of the parameters up to order $p$, the resulting interpolation function becomes

$$
\widehat{A}(\mathbf{s})=\sum_{i=1}^{M} \widehat{B}_{i} \Phi\left(\left\|\mathbf{s}-\mathbf{s}_{i}\right\|\right)+\sum_{i=1}^{K} \widehat{C}_{i} p_{i}(\mathbf{s}) .
$$

Here, $\Phi$ is given by (7) and $\left\{p_{1}, \ldots, p_{K}\right\}$ is a basis for polynomials of $\mathbf{s}$ up to degree $p$. If the function being interpolated is sufficiently smooth, it can be shown that this approximation converges as the distance between the sample points goes to zero [4].

The interpolation coefficients are determined as follows. First, the matrices

$$
\begin{aligned}
R_{m n} & =\Phi\left(\left\|\mathbf{s}_{m}-\mathbf{s}_{n}\right\|\right) \\
P & =\left(\begin{array}{ccc}
p_{1}\left(\mathbf{s}_{1}\right) & \cdots & p_{K}\left(\mathbf{s}_{1}\right) \\
\vdots & \ddots & \vdots \\
p_{1}\left(\mathbf{s}_{M}\right) & \cdots & p_{K}\left(\mathbf{s}_{M}\right)
\end{array}\right)
\end{aligned}
$$

are computed. Then the system

$$
\left(\begin{array}{cc}
R & P \\
P^{T} & 0
\end{array}\right)\left[\begin{array}{l}
(\widehat{B})_{i j} \\
(\widehat{C})_{i j}
\end{array}\right]=\left[\begin{array}{c}
(\tilde{A})_{i j} \\
\mathbf{0}
\end{array}\right]
$$

is solved. In the right-hand side and solution vectors, the notation $(X)_{i j}$ means a vector containing the $(i, j)$ entries of the $X$ matrix. These coefficients must be recalculated every time a sample point is added, since the $R, P$, and $\tilde{A}$ matrices will all change

\section{SAMPLING}

The choice for the projection matrix $V$ in the definition

$$
\tilde{A}=V^{H} A V
$$

is now straightforward. Since the coefficients of (8) have been chosen so as to satisfy the interpolation property (4), it is natural that we also be able to recover the exact solution $\mathrm{x}$ at the samples points. This in turn requires

$$
\text { colspace } V=\operatorname{span}\left\{\mathbf{x}\left(\mathbf{s}_{0}\right), \mathbf{x}\left(\mathbf{s}_{1}\right), \ldots, \mathbf{x}\left(\mathbf{s}_{K}\right)\right\} .
$$

For numerical stability, it is desirable for $V$ to be unitary. This can be achieved by appending each new solution vector to $V$ and performing modified Gram-Schmidt orthogonalization.

In choosing the sample points, we are motivated by the desire to find the point where the error is maximized. One possibility is to choose a large number of points, either randomly or on a grid, and select the one with largest estimated error. This approach has been applied in [5] but is suboptimal, since it will likely give a point near, but not at, a maximum. Thus, we follow the lead of [6] and apply an optimization algorithm to the error estimator, in order to find a point where it is maximized. That is, given an error estimator $E E(\mathbf{s})$, we aim to solve the problem

$$
\underset{\mathbf{s}_{\min } \leq \mathbf{s} \leq \mathbf{s}_{\max }}{\arg \max } E E(\mathbf{s}) .
$$

Notably, the search is not restricted to a finite set of points, but rather ranges across the entire, continuous parameter space. We use a Newton-like method, the interior trust region method [7], [8].

\section{A. Optimized Sampling}

In order to find the maximum error, the interior trust region method [7] is used. This process begins by considering a quadratic function closely related to the second-order Taylor series approximation of the error estimator,

$$
\begin{aligned}
\psi(\mathbf{p}) & =\mathbf{g}^{T} \mathbf{p}+\frac{1}{2} \mathbf{p}^{T}(H+C) \mathbf{p} \\
& \approx E E\left(\mathbf{s}_{0}+\mathbf{p}\right)-E E\left(\mathbf{s}_{0}\right)+\frac{1}{2} \mathbf{p}^{T} C \mathbf{p},
\end{aligned}
$$

where $\mathbf{g}$ and $H$ are the gradient and Hessian, and $\mathbf{s}_{0}$ is the current approximation of the maximum. The $C$ matrix depends on the parameter space boundary and biases the process against crossing nearby boundaries. This approximation is 
used to find two search directions. One, the Newton direction, is the solution to

$$
\underset{\|D \mathbf{p}\|<\Delta}{\arg \min } \psi(\mathbf{p}) .
$$

The area $\|D \mathbf{p}\|<\Delta$ is called the trust region. The other direction is $D^{-2} \mathbf{g}$. In both cases, $D$ is a scaling matrix that again biases the directions towards remaining feasible. Next, a line search is performed along both directions to determine which one increases $\psi$ the most, while remaining inside the parameter space and the trust region. This is the candidate step $\Delta \mathbf{s}$.

Finally, $\psi(\Delta \mathbf{s})$ is compared to the actual change in the error estimator using the candidate step. If the two are sufficiently close, then we know that $\psi$ is a good approximation and accept the candidate step. Otherwise, it is rejected and $\Delta$ is decreased. That is, the next candidate step is restricted to a region closer to the Taylor series expansion point, where $\psi$ will be more accurate. This process continues until $\left\|D^{-2} \mathbf{g}\right\|<\epsilon_{\text {opt }}$.

\section{ERROR ESTIMATOR}

Lastly, the error estimator is considered. It is here that numerous variations are possible. We suggest an estimator based on the difference between the MOR solution and a polynomial interpolation of the solution, i.e.,

$$
E E(\mathbf{s})=\frac{\left\|\widehat{A}^{-1}(\mathbf{s}) V^{H} \mathbf{b}(\mathbf{s})-\sum_{i=1}^{M} \mathbf{x}_{i} q_{i}(\mathbf{s})\right\|}{\left\|\widehat{A}^{-1}(\mathbf{s}) V^{H} \mathbf{b}(\mathbf{s})\right\|} .
$$

Because the number of sample points $M$ increases one at a time, the polynomial basis $\left\{q_{i}\right\}$ will rarely be complete. Therefore, the method of [9] is used to generate a basis regardless of the dimension of $\mathbf{s}$ of the number of sample points. Although most of the terms in (18) are known analytically, $\mathbf{b}$ is not. Therefore, the error estimator's derivatives, needed for the previously-described optimization algorithm, cannot be exactly calculated. Although one could make the entire expression a known function by replacing $\mathbf{b}$ with an interpolation, we have found finite differences to be sufficient.

The reasoning behind this estimator is that each initially provide its own, different approximation to the result. As more samples are added, each will converge, so that the difference between them gives an estimate of how far each is from the true solution.

\section{EXAMPLES}

The following results use the unoptimized sampling strategy described in [5], i.e., the next sample point is taken from a finite list of points distributed throughout the parameter space. Results from the optimized method are in progress and will be discussed at the conference.

\section{A. Patch Antenna}

Our first example is a patch antenna analysed using the FEM-based MOR method of [1]. The antenna is sandwiched between two dielectric layers, $18 \mathrm{~mm}$ wide by $18 \mathrm{~mm}$ long, as shown in Fig. 1(a). The parameters are the frequency ( $f=$ $3-8 \mathrm{GHz})$ and the dielectric constants of the top $\left(\varepsilon_{r 1}=1-7\right)$



(a)

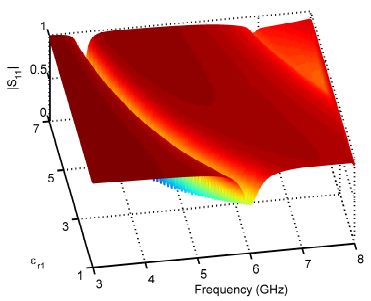

(c)

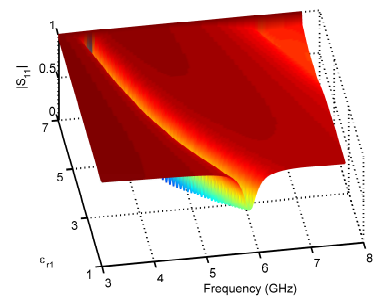

(b)



(d)
Fig. 1. $S_{11}$ of patch antenna with $\varepsilon_{r 2}=3$. (a) antenna geometry; (b) adaptive MOR, $\tau=0.1$; (c) adaptive MOR, $\tau=0.01$; (c) method in [1].

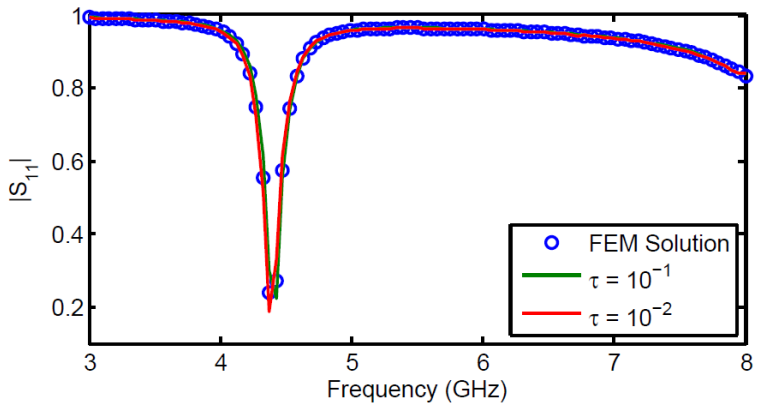

(a) Comparison with full-wave solution

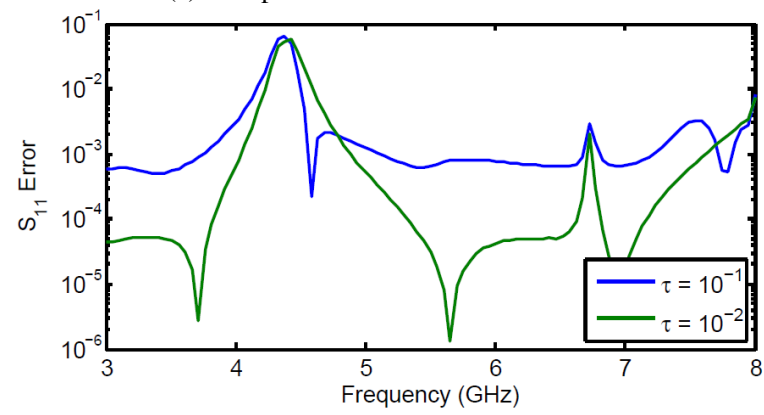

(b) Error

Fig. 2. Convergence of adaptive MOR.

and bottom $\left(\varepsilon_{r 2}=1-7\right)$ layers. The problem has 84,718 DOFs and takes $33 \mathrm{~s}$ to solve using a parallel FEM solver and 16 CPUs.

Figure 1(b)-(d) shows the result of our adaptive MOR with two different tolerances, compared to the MOR solution in [1]. It can be seen that the first, crude model indeed captures the major features of the response. Further, tightening the adaptive MOR tolerance brings out additional detail in the solution. This convergence is confirmed in Fig. 2, which shows the $S_{11}$ error for the two models. With only a few exceptions, the $\tau=10^{-2}$ model is significantly more accurate than the 
TABLE I

COMPUTATIONAL RESULTS FOR PATCH ANTENNA AND CIRCUIT BOARD EXAMPLES.

\begin{tabular}{l|cc|c|c} 
& \multicolumn{3}{|c|}{ Patch Antenna } & Circuit Board \\
& Tolerance & Tolerance & Farle \& & Tolerance \\
& $\tau=0.1$ & $\tau=0.01$ & Dyczij-Edlinger & $\tau=0.1$ \\
\hline Model Dimension & 22 & 39 & 165 & 10 \\
Model Creation Time (hh:mm) & $1: 01$ & $2: 48$ & $1: 17$ & $0: 27$ \\
Model Evaluation Time (ms) & 60.1 & 114 & 13.7 & 2.1
\end{tabular}

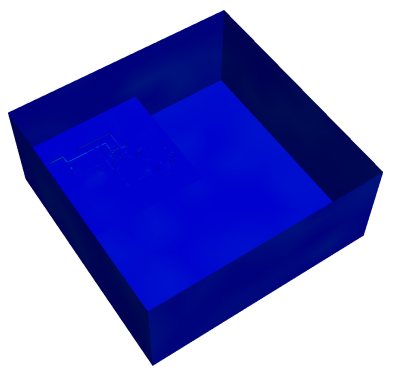

(a)

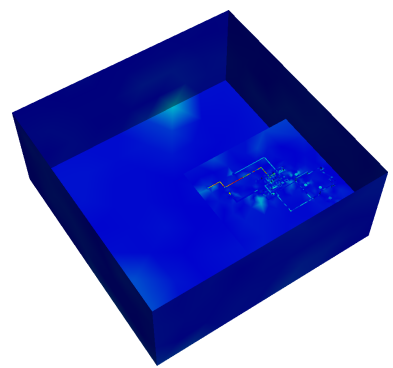

(b)
Fig. 3. Movable circuit board inside metallic enclosure. (a) $\mathrm{CB}$ in upper-left corner; (b) $\mathrm{CB}$ in lower-right corner.

$\tau=10^{-1}$ model. Both models show considerable error around the resonance, and this is likely due to the fact that only certain discrete locations are available for sampling.

Computational statistics are shown in Table I. They show that by sacrificing some of the extreme accuracy of traditional MOR methods, it is possible to gain a significant reduction in the model dimension (i.e., the number of full-wave solutions). And although the computation times compare less favorably, this can be viewed as an acceptable trade-off for the increased automation and generality.

\section{B. Circuit in Metallic Box}

Our second example consists of a circuit board inside a metallic box with an aperture, as shown in Fig. 3(a). The enclosure is $15 \mathrm{~cm} \times 15 \mathrm{~cm} \times 7 \mathrm{~cm}$, and the board is free to move in the forward, back, left, and right directions. A plane wave is incident on the box from above, and we are interested in the induced current on the circuit board as a function of its location inside the box. Despite its relative simplicity, this is a challenging problem for MOR methods. The EFIE is well-suited for this situation because the box and $\mathrm{CB}$, being disconnected, can be moved arbitrarily without changing the number of unknowns. Recent MoM methods, however, only consider frequency as a parameter [10].

Our method easily handles this problem, as Fig. 3 shows. At $2 \mathrm{GHz}$, the surface discretization has 4,122 unknowns. We wish to emphasize at this point our earlier assumption that only matrix solves, matrix-vector multiplications, and righthand-side evaluations are used. The original EFIE program was modified only to do these operations and is in no way specialized for this particular problem. Instead, a few small Python scripts and helper programs are needed to move the CB mesh coordinates as needed. Thus, not only is the method adaptable to a wide array of problems, it is also significantly easier to integrate with existing EM codes.

\section{CONCLUSION}

An adaptive MOR algorithm has been described that uses radial basis functions to interpolate the reduced order matrix, making the method applicable to a large range of EM solvers. Numerical examples show that the method is competative with other MOR approaches and is applicable to a variety of problems.

\section{REFERENCES}

[1] O. Farle and R. Dyczij-Edlinger, "Numerically stable moment matching for linear systems parameterized by polynomials in multiple variables with application to finite element models of microwave structures," IEEE Trans. Antennas Propag., vol. 58, no. 11, pp. 3675-3684, Nov. 2010.

[2] R. D. Slone, J.-F. Lee, and R. Lee, "Automating multipoint galerkin awe for a fem fast frequency sweep," IEEE Trans. Magn., vol. 38, no. 2, pp. 637-640, Mar. 2002.

[3] M. D. Buhmann, "Radial basis functions," Acta Numerica, vol. 9, pp. $1-38,2000$.

[4] R. Schaback, "Approximation by radial basis fucntions with finitely many centers," Constr. Approx., vol. 12, no. 3, pp. 331-340, 1996.

[5] M. B. Stephanson and J.-F. Lee, "Adaptive, black-box model order reduction using radial basis fucntions," in Proc. International Review of Progress in Applied Computational Electromagnetics, Williamsburg, VA, Mar. 2011, accepted for presentation.

[6] T. Bui-Thanh, K. Willcox, and O. Ghattas, "Model reduction for largescale systems with high-dimensional parametric input space," SIAM J. Sci. Comput., vol. 30, no. 6, pp. 3270-3288, 2008.

[7] T. F. Colemen and Y. Li, "An interior trust region approach for nonlinear minimization subject to bounds," SIAM J. Optimization, vol. 6, no. 2, pp. 418-445, May 1996.

[8] J. J. Moré and D. C. Sorensen, "Computing a trust region step," SIAM J. Sci. Stat. Comput., vol. 4, no. 3, pp. 553-572, Sep. 1983.

[9] C. de Boor and A. Ron, "Computational aspects of polynomial interpolation in several variables," Math. of Comput., vol. 58, no. 198, pp. 705-727, Apr. 1992.

[10] J. Yeo and R. Mittra, "An algorithm for interpolating the frequency variations of method-of-moments matrices arising int he analysis of planer microstrip structures," IEEE Trans. Microw. Theory Tech., vol. 51, no. 3, pp. 1018-1025, Mar. 2003. 